\title{
Time and temperature effects on Poisson's ratio of poly(butylene terephthalate)
}

\author{
S. Pandini ${ }^{*}$, A. Pegoretti ${ }^{2}$ \\ ${ }^{1}$ Department of Mechanical and Industrial Engineering and INSTM Research Unit, University of Brescia, via Branze 38, \\ 25133, Brescia, Italy \\ ${ }^{2}$ Department of Materials Engineering and Industrial Technologies and INSTM Research Unit, University of Trento, \\ via Mesiano 77, 38123, Trento, Italy
}

Received 26 November 2010; accepted in revised form 2 February 2011

\begin{abstract}
The viscoelastic nature of the Poisson's ratio of a semicrystalline poly (butylene terephthalate) is highlighted by investigating its dependence on time, temperature and strain rate, under two types of loading conditions: i) constant deformation rate tests, in which the transverse strain is measured in tensile ramps at various temperatures and at two strain rates; and ii) constant deformation tests, in which, under a constant axial deformation, the transverse strain is measured as a function of time in isothermal experiments performed at various temperatures. In both testing configurations, axial and transverse deformations are measured by means of a biaxial contact extensometer, and a correction procedure is adopted in order to compensate the lateral penetration of the extensometer knives. Poisson's ratio displays the typical features of a retardation function, increasing with time and temperature, and decreasing with strain rate. This behaviour has been compared to that of simultaneously measured relaxation modulus.
\end{abstract}

Keywords: material testing, mechanical properties, viscoelasticity, Poisson's ratio

\section{Introduction}

In the infinitesimal deformation of an idealized purely elastic material, Poisson's ratio is a material constant, defined for an uniaxial stress state as the negative ratio between the deformation normal to the loading axis and the axial imposed strain.

For viscoelastic solids, as in the case of polymers, the lateral contraction coupled to the infinitesimal axial extension exhibits a time and temperature dependent behaviour. The Poisson's ratio of viscoelastic materials cannot thus be regarded as a constant parameter, but as a time-dependent material function, deserving a proper description that encompasses the dependence on time, or equivalently on frequency and strain rate, on temperature and on materials mechanical history.
Poisson's ratio of polymeric materials can thus be thought in the framework of linear viscoelasticity as one of the various viscoelastic response functions, but its determination can involve more complexity [1-3]. In fact, relaxation moduli and creep compliances are usually determined in conditions that allow an easy description of a time dependent correlation between stress and strain (i.e. stress relaxation tests, in which the axial strain is kept constant, and creep tests, in which the stress is constant). On the other hand, in the case of Poisson's ratio, a time dependent correlation has to be established between two strains and a stress generating one of them. As it was underlined by theoretical studies [1, 3-4], when investigating Poisson's ratio time dependence, it is of primary importance to provide a proper

\footnotetext{
${ }^{*}$ Corresponding author, e-mail: stefano.pandini@ing.unibs.it
} (c) BME-PT 
definition of Poisson's ratio as a viscoelastic function and a clear indication of its evaluation conditions. The importance of this aspect is often neglected [1], although the obtained results are shown to depend on testing conditions [3].

In spite of the time dependent behaviour of polymers, and due to considerable experimental difficulties, Poisson's ratio of polymeric materials is often assumed as a constant, or, occasionally, defined for values corresponding to glassy and rubbery equilibrium conditions. In both cases its viscoelastic nature is completely neglected [1]. Nevertheless, it was shown that only in few cases the assumption of a constant Poisson's ratio can be reasonably considered valid [2, 4], and that neglecting its time and temperature dependence may lead to predicting models markedly deviating from the experimental observations [5-7]. More detailed information on the viscoelastic behaviour of Poisson's ratio is therefore important not only in principle [8-10], but also for practical reasons. In fact, it would allow us to interconvert material functions [1], to refine computational methods [2], to reduce the number of required experimental parameters to fit analytical models [8], to better estimate the effects of the so called Poisson's strains, originating from misfits in Poisson's ratio of joined materials [1].

So far, only few trials have been attempted to comprehensively measure and describe the viscoelastic nature of Poisson's ratio. In a review article, Tschoegl et al. [1] summarize the main results concerning the measurement and the prediction of viscoelastic Poisson's ratio, and also give a critical perspective on the problems regarding its determination.

A viscoelastic Poisson's ratio can be experimentally evaluated following two procedures; a so called 'direct' measurement, which involves the measurement of the transverse and axial deformations of the tested specimen; and a so called 'indirect' measurement, where, on the basis of the linear viscoelasticity, Poisson's ratio is calculated from the measurement of two other material's time dependent parameters. This latter approach has been proven to be a difficult path, since requiring a very accurate and precise knowledge of the time dependence of the starting functions [11].
Direct measurements of Poisson's ratio has been carried out on polymeric materials by different types of experiments, in which the axial deformation was applied through a constant rate loading ramp [12-15], under a constant load (creep) [13, 16-17], under constant strain (stress relaxation) $[10,14,18]$, or under sinusoidal loading-unloading (dynamicmechanical analysis) [19-21]. An accurate measurement of the transverse strains is of crucial importance and various techniques have been employed, such as optical methods (i.e. Moirè interferometry $[10,17-18]$ and video extensometry $[13,16])$, bonded strain gages [19-20] and biaxial contact extensometers [14-15, 21].

Poisson's ratio of polymeric materials is generally reported as increasing with time ( $\mathrm{Lu}$ et al. on poly (methylmethacrylate) (PMMA) [10], di Landro and Pegoraro on poly (etherimide) (PEI) [18]), temperature (Tcharkhtchi on epoxy resins [15]), and strain (Steinberger et al. on polypropylene (PP) [13]; Litt and Torp on polycarbonate (PC) [22]). In a work on epoxy resins we have also reported Poisson's ratio as increasing with time, temperature and strain, and decreasing with strain rate [14]. Moreover, a decreasing trend has been reported for the complex Poisson's ratio with frequency under dynamic conditions (Kästner and Pohl on PMMA [23]; Caracciolo and coworkers on poly(vinylchloride) (PVC) [1920]; Arzoumanidis and Liechti on a neat urethane adhesive (Ashland) [21]; Pritz, by indirect methodology, on rubbery materials and PMMA [24]).

The results are consistent to what predicted by analytical approaches, which describe the viscoelastic Poisson's ratio following an increasing trend with time $[2,4,8]$, featuring the typical trend of a retardation process that can be approached as a transverse creep compliance [25]. Nevertheless, in his studies on auxeticity, Lakes depicts a vaster framework and suggests that for structured materials a monotonic dependence of Poisson's ratio cannot be assumed a priori [26].

In the present paper, an experimental investigation of the viscoelastic features of the Poisson's ratio of a semicrystalline material, poly (butylene terephthalate), PBT, is attempted under constant strain rate and constant strain conditions. The measurements are carried out by means of a biaxial contact extensometer and a procedure is proposed in order 
to minimize the effects of the extensometer on the measured transverse strains. Aim of the work is to provide a description of the effects of time, strain rate and temperature on the viscoelastic Poisson's ratio.

\section{Experimental}

\subsection{Material and preliminary characterization}

Injection moulded PBT dumb-bell specimens (ISO 527 type 1A; gauge length: $80 \mathrm{~mm}$; cross-section: $4 \times 10 \mathrm{~mm}$ ) were kindly supplied by Radici Novacips SpA (Villa d'Ogna, Bergamo, Italy). All specimens were treated for $3 \mathrm{~h}$ at $190^{\circ} \mathrm{C}$ under vacuum and slowly cooled in the oven in order to release thermal stresses and uniform the thermal history. In order to detect possible anisotropic effects on the mechanical behaviour of the tensile bars, cubic specimens were machined from the dumb-bell central region and tested under compression along the $x, y$ and $z$ axes (i.e. through specimen length, width and thickness, respectively). On an interval of compressive strains ranging up to 0.6 no significant dependence of the mechanical behaviour on the loading directions was detected.

Differential scanning calorimetry (DSC) analyses were carried out by a Mettler DSC-30 calorimeter (Mettler Toledo, Zürich, Switzerland) at a heating rate of $10^{\circ} \mathrm{C} / \mathrm{min}$ in a nitrogen flux of about $100 \mathrm{ml} / \mathrm{min}$. Tests were performed on specimens of about $15 \mathrm{mg}$ machined from the gauge length of the dumb-bell specimens. From DSC measurements a glass transition temperature $\left(T_{\mathrm{g}}\right)$ of $47^{\circ} \mathrm{C}$, a melting point $\left(T_{\mathrm{m}}\right)$ of $220^{\circ} \mathrm{C}$ and a crystallinity content $\left(X_{\mathrm{c}}\right)$ of $38 \%$ were determined. The cristallinity percentage was assessed by integrating the normalized area under the melting endothermal peak and rating the heat involved to the reference value of $100 \%$ crystalline polymer, corresponding to $145 \mathrm{~J} / \mathrm{g}$ [27].

Dynamic mechanical thermal analysis (DMTA) experiments were performed by means of an MkII Polymer Laboratories DMTA testing-machine. Tests were carried out under bending mode on rectangular bars $(12 \times 5 \times 2 \mathrm{~mm})$ machined from the gauge length of a dumb-bell specimen at the test frequencies of $0.3,1,3,10,30 \mathrm{~Hz}$.

The region of linear viscoelastic behaviour was evidenced on isochronous stress-strain curves obtained by stress relaxation tests performed at axial strain levels in the range from 0.001 to 0.02 . Mechanical tests were performed on PBT dumb-bell specimens by means of an electromechanical testing machine (Instron, model 4502, Instron, Norwood, Massachusetts, USA), controlling the level of strain by an axial extensometer (Instron, model 2640, Instron, Norwood, Massachusetts, USA). The deformation was applied at a constant crosshead speed (corresponding to a strain rate of $=0.003 \mathrm{~s}^{-1}$ ) and then kept constant for at least $30 \mathrm{~min}$. The tests were performed at $30^{\circ} \mathrm{C}$ and at $70^{\circ} \mathrm{C}$ (i.e. below and above the glass transition temperature) in a thermostatic chamber (Instron, model 3199, Instron, Norwood, Massachusetts, USA ), permitting a temperature control within $\pm 1^{\circ} \mathrm{C}$.

\subsection{Viscoelastic Poisson's ratio evaluation}

The dependence of Poisson's ratio on time, temperature and strain rate was experimentally evaluated under various testing conditions. In particular, as schematically represented in Figure 1, two different loading histories were investigated: in constant deformation rate (CDR) tests the specimen was subjected to a tensile ramp at a fixed strain rate; in constant deformation tests (REL) a constant tensile axial deformation was applied, such as in stress relaxation tests. In both CDR and REL tests, the input (axial deformation, $\varepsilon_{\mathrm{AX}}$ ) and output (transverse deformation, $\varepsilon_{\text {TRANS }}$, and stress, $\sigma$ ) variables were simultaneously recorded as a function of time. The experiments were performed with an Instron 4502 electromechanical testing machine (see above), operating in displacement control. The tests were performed in a thermostatic chamber (see above) at various temperatures between 20 and $100^{\circ} \mathrm{C}$.

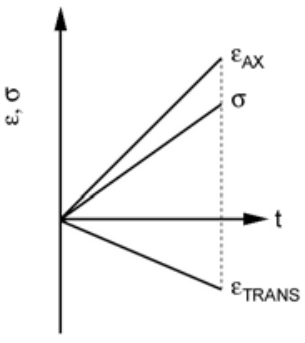

a) CDR experiment

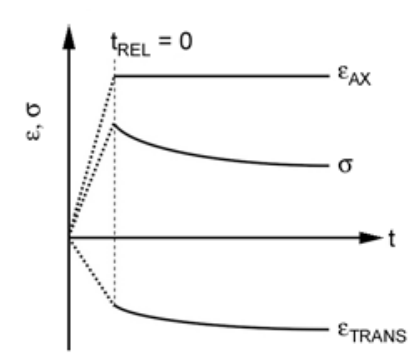

b) REL experiment
Figure 1. Schematic representation of input (axial deformation, $\varepsilon_{\mathrm{AX}}$ ) and output (stress, $\sigma$; transverse deformation, $\varepsilon_{\text {TRANS }}$ ) variables in a) constant deformation rate (CDR) experiments and b) constant deformation (REL) experiments 
The axial and transverse deformations were evaluated by means of a biaxial clip-on extensometer (Instron, model 2620-613, Instron, Norwood, Massachusetts, USA) mounted on the specimen, as represented in Figure 2. The extensometer is a selfstanding clip-on device, weighing about $300 \mathrm{~g}$, which is symmetrically distributed on the specimen, thanks to the peculiar shape of the device. No other supports are required to sustain the device, since visual inspection during the test has never detected distortion of the specimen at the temperatures explored. In order to assure self-standing and gripping, the extensometer exerts on the lateral sides of the specimen a certain force, quantified by the producer to a nominal value of $22 \mathrm{~N}$ [http:// www.instron.com/wa/library/StreamFile.aspx? $\mathrm{doc}=$ 290]. This generates a local transverse stress of about $0.5 \mathrm{MPa}$, roughly evaluated by dividing the gripping force over the gauge side cross-section defined by the specimen thickness multiplied for the distance between the knives. The value is at least one order of magnitude lower than the lowest axial stress measured in constant deformation experiments, and the effect of this transverse stress is considered to have a minor contribution to the overall stress state with respect to the axial tensile stress, but leads anyway to a continuous penetration of the extensometer knives in the specimen (creep penetration). The transverse displacement measured during the tests originates thus both from the material contraction (ruled by the Poisson's ratio) and from the lateral creep exerted by the extensometer. In order to compensate the data for this undesired

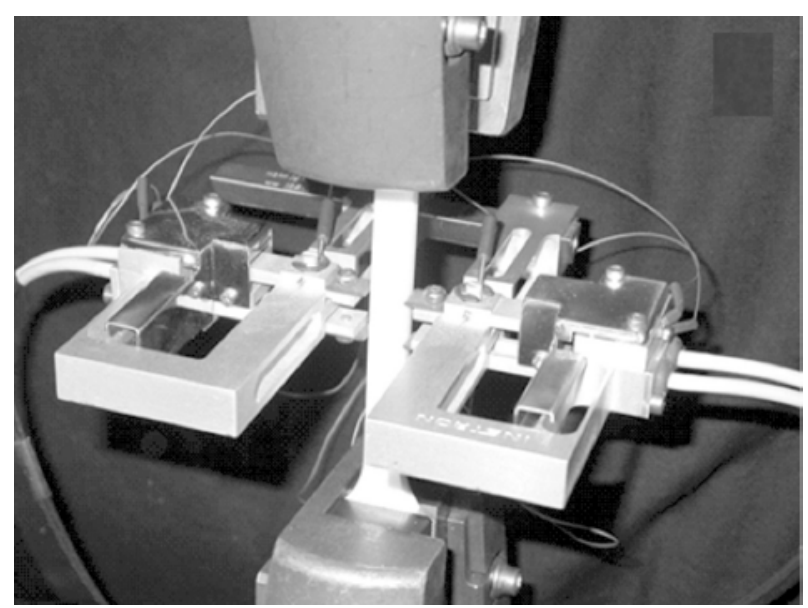

Figure 2. Experimental set-up employed for the Poisson's ratio measurement, in adoption of a clip-on biaxial extensometer (Instron mod. 2620) mounted on a PBT dumb-bell specimen effect, the amount of creep penetration of the extensometer was evaluated separately on undeformed specimens as a function of time at various temperatures and then subtracted from the total transverse displacement recorded.

The longitudinal deformation, $\varepsilon_{\mathrm{AX}}$, was evaluated as shown by Equation (1):

$\varepsilon_{\mathrm{AX}}=\frac{\Delta L_{\mathrm{AX}}}{L_{\mathrm{AX}, 0}}$

where $\Delta L_{\mathrm{AX}}$ represents the axial displacement measured by the extensometer and $L_{\mathrm{AX}, 0}$ the longitudinal initial gauge length $(12.5 \mathrm{~mm})$.

The transverse deformation, $\varepsilon_{\text {TRANS }}$, has been measured across the specimen width, and evaluated as shown by Equation (2):

$$
\varepsilon_{\text {TRANS }}=\frac{\Delta L_{\text {TRANS }}-\Delta L_{\text {PENETRATION }}}{L_{\text {TRANS }, 0}}
$$

where, for a given time, $\Delta L_{\text {TRANS }}$ represents the transverse displacement recorded by the extensometer and $\Delta L_{\text {PENETRATION }}$ the extensometer penetration, while $L_{\text {TRANS }, 0}$ is the specimen initial width.

The transverse deformation was measured across the width of the specimen, since permitting to deal with larger transverse displacement signals with respect to those measured when the transverse deformation measured across the specimen thickness. This testing geometry helps in minimizing the contribution of dimensional defects of the specimens and of the imprecision in the compensation for the transverse extensometer penetration. The Poisson's ratios described in this paper always refer to such testing direction, although, due to the overall material isotropy, it is expected that very similar results could be obtained if lateral contraction would be measured through the thickness.

In constant deformation rate (CDR) tests the specimen was subjected to a tensile loading ramp at a constant strain rate. Aim of the CDR tests is to provide a description of the effects of temperature and strain rate on the Poisson's ratio evaluated at short times and at small axial strains. We will refer to the values measured under these testing conditions as Poisson's ratio, but still bearing in mind the indication of Hilton and Yi [4] and Tschoegl et al. [1], who prefer the term lateral contraction ratio over Poisson's ratio, for the data measured in ramp tests. The tests were performed at two crosshead speeds, 
corresponding to strain rates of 0.003 and $0.05 \mathrm{~s}^{-1}$, and at various temperatures, in the range between -20 and $100^{\circ} \mathrm{C}$, while the maximum strain never exceeded 0.01. The Poisson's ratio values measured under these experimental conditions will be labelled as $v_{\mathrm{CDR}}$, and calculated by Equation (3) as the negative ratio between the slopes of the transversal deformation and axial deformation curves as function of the applied load, $F$ :

$\nu_{\mathrm{CDR}}=-\frac{\frac{\mathrm{d} \varepsilon_{\mathrm{TRANS}}}{\mathrm{d} F}}{\frac{\mathrm{d} \varepsilon_{\mathrm{AX}}}{\mathrm{d} F}}$

on the region of linear proportionality between stress and strain. The evaluation takes the advantage of the linear dependence of the axial and transverse deformations on the applied load on an adequately large portion of the axial strain values (i.e. for $\varepsilon_{\mathrm{AX}}$ at least up to 0.03 ), and is proposed accordingly with standardized procedures for the evaluation of short-time tensile properties of plastics (ASTM D 638). Further, it has to be remarked that, when force and the two strains can be described by functions linearly increasing with time from a zero value at $t=0$, the above formula provides results coincident with those that can be obtained by applying Equation (4) proposed by Tschoegl et al. [1] for the evaluation of the lateral contraction ratio in constant rate of strain measurements:

$\nu(t)=\frac{1}{t} \int_{0}^{t} \nu_{\text {rate }}(u) \mathrm{d} u$

where $v_{\text {rate }}$ can be defined by Equation (5):

$\nu_{\text {rate }}=-\frac{\frac{\mathrm{d} \varepsilon_{\text {TRANS }}}{\mathrm{d} t}}{\frac{\mathrm{d} \varepsilon_{\mathrm{AX}}}{\mathrm{d} t}}=-\frac{\frac{\mathrm{d} \varepsilon_{\mathrm{TRANS}}}{\mathrm{d} F}}{\frac{\mathrm{d} \varepsilon_{\mathrm{AX}}}{\mathrm{d} F}}$

and, for a given strain rate, it displays a constant value on the linear stress $v s$. strain region.

Young's modulus was also measured under the same test conditions.

Constant deformation tests (REL) were performed applying a constant deformation $\left(\varepsilon_{\mathrm{AX}, \mathrm{REL}}\right)$ for at least $30 \mathrm{~min}$ and measuring the transverse deformation as a function of time. These tests were carried out for two values of the constant axial strain, $\varepsilon_{\mathrm{AX}, \mathrm{REL}}=0.005$ and 0.010 , applied at a strain rate of
$0.003 \mathrm{~s}^{-1}$, and at various temperatures in the range between 30 and $60^{\circ} \mathrm{C}$. The time dependence of Poisson's ratio was assessed by measuring the changes in the specimen width after the axial deformation was applied and maintained constant. This type of test follows the typical methodology of a stress relaxation test, and thus the Poisson's ratio measured in these conditions is labelled as $v_{\text {REL }}$, and evaluated according to Equation (6):

$\nu_{\mathrm{REL}}=-\frac{\varepsilon_{\mathrm{TRANS}}(t)}{\varepsilon_{\mathrm{AX}, \mathrm{REL}}}$

where $\varepsilon_{\text {TRANS }}$ is the time-dependent transverse deformation measured for the applied longitudinal deformation $\varepsilon_{\mathrm{AX}, \mathrm{REL}}$. Since the axial deformation is constant in time, this type of test configuration permits an easy but rigorous calculation of the viscoelastic Poisson's ratio, thus avoiding the otherwise complicated mathematical treatments required for other deformational histories and involving Laplace transforms between viscoelastic variables [1]. Relaxation modulus was concurrently measured in this part of the test and will be reported as $E_{\mathrm{REL}}$. The results were then plotted as a function of $t_{\mathrm{REL}}=$ $t-t_{0}$, where $t$ represents the test time and $t_{0}$ the time at which the constant deformation for the relaxation experiment is applied.

\section{Results and discussion}

\subsection{Preliminary material characterization}

Figure 3 reports the traces of the storage modulus $\left(E^{\prime}\right)$ and $\operatorname{loss}$ factor $(\tan \delta)$ as a function of temperature at various frequencies. The figure shows a glass transition temperature region between 45 and $70^{\circ} \mathrm{C}$, depending on the frequency, as evaluated in

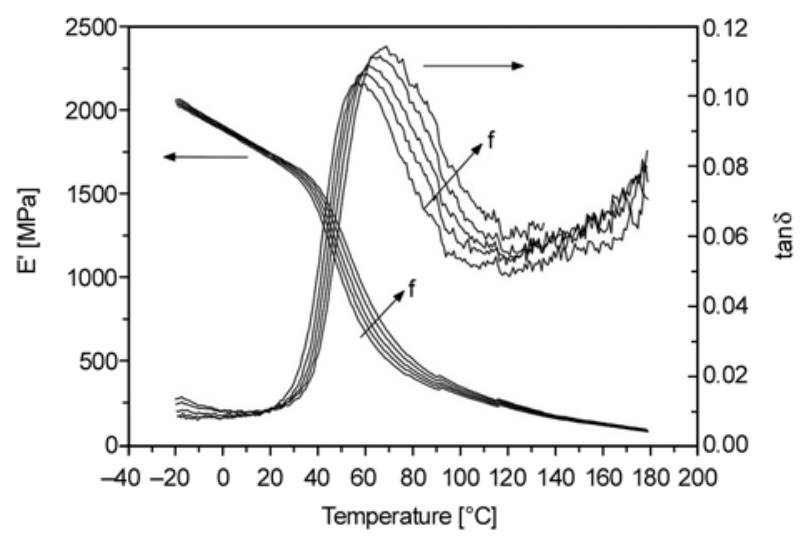

Figure 3. $E^{\prime}$ and $\tan \delta$ traces from the DMTA test performed on PBT at various frequencies $(f=0.3,1,3,10$, $30 \mathrm{~Hz}$ ) 


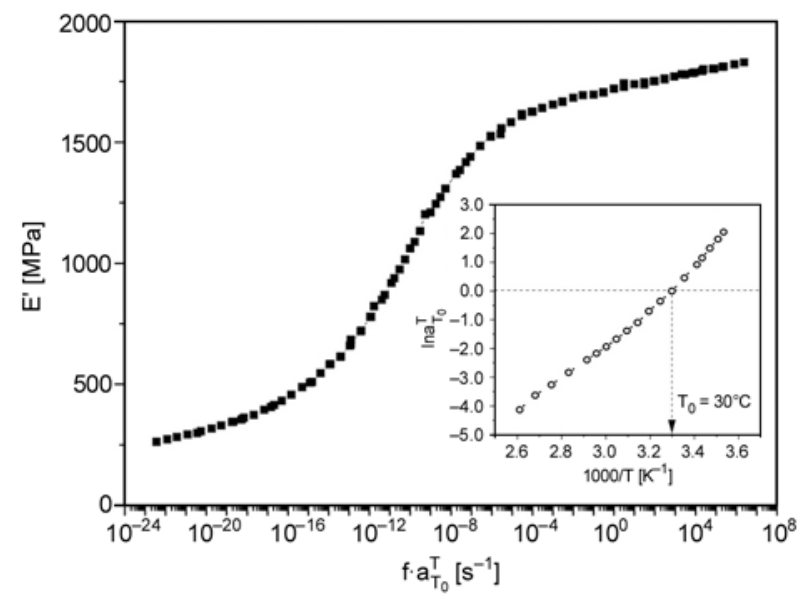

Figure 4. $E^{\prime}$ master curve as a function of reduced frequency at a reference temperature, $T_{0}=30^{\circ} \mathrm{C}$; insert: shift factors evaluated by best superposition of isothermal $E^{\prime} v s$. frequency curves

correspondence to the region where the storage modulus traces present an inflection point and the $\tan \delta$ traces show their maximum. According to a time-temperature reduction scheme, isothermal $E^{\prime}$ $v s$. frequency curves enabled us to build a storage modulus master curve, represented in Figure 4 for a reference temperature $T_{0}=30^{\circ} \mathrm{C}$. The logarithm of the shift factors, $a_{\mathrm{T}_{0}}^{\mathrm{T}}$, adopted for the master curve construction are presented as an insert in Figure 4, as a function of $1000 / T$, where $T$ is the absolute temperature. The master curve indicates that the transition zone of the storage modulus spans over a frequency range of 7-8 decades, ranging i.e. from about $10^{-15} \mathrm{~Hz}$ up to about $10^{-8} \mathrm{~Hz}$ at this reference temperature.

The extent of the linear viscoelastic region was assessed on isochronous stress-strain curves obtained from stress relaxation tests performed at 30 and at

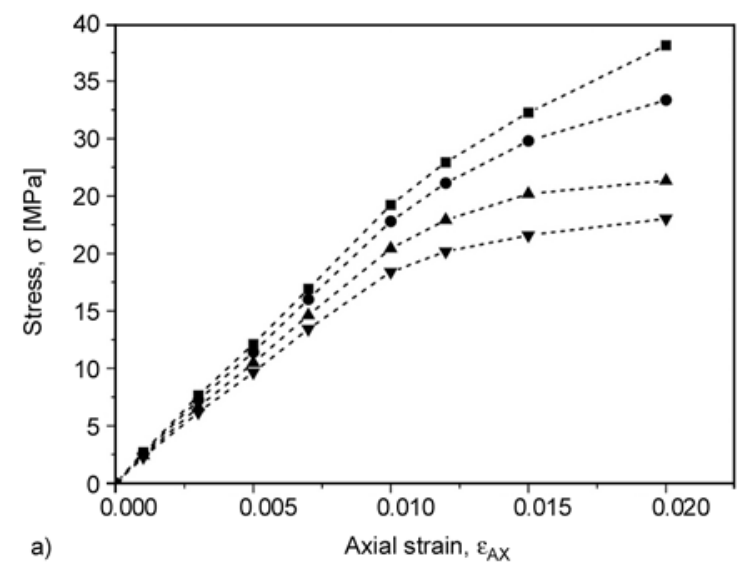

$70^{\circ} \mathrm{C}$, i.e. below and above the glass transition temperature. The isochronous curves for these two temperatures are reported at various instants in Figure $5 \mathrm{a}$ and $\mathrm{b}$, respectively. Independently from time, the curves at $30^{\circ} \mathrm{C}$ show a linear trend for axial deformations up to 0.01 , whereas at higher deformations an evident deviation from linearity can be detected. On the other hand, a clear threshold value for the linear viscoelastic behaviour is more difficult to be identified in the experiments performed at $70^{\circ} \mathrm{C}$. Anyway, the results seem to indicate that at this temperature the linear viscoelastic region extends up to an axial strain level between 0.005 and 0.01 . In consideration of these results the evaluation of Poisson's ratio in constant deformation experiments was carried out both at an axial strain $\varepsilon_{\mathrm{AX}, \mathrm{REL}}=0.005$ and at $\varepsilon_{\mathrm{AX}, \mathrm{REL}}=0.01$.

\subsection{Viscoelastic Poisson's ratio effect and correction of the transverse displacement}

Experimental input and output of CDR experiments are reported in Figures $6 a$ and b, as measured in tests performed at a strain rate of $0.003 \mathrm{~s}^{-1}$ at some of the investigated temperatures. Figure 6a represents the time evolution of the stress during the tensile ramp test and its well-known dependence on temperature. In Figure $6 \mathrm{~b}$ the reading of the transverse extensometer is reported in terms of an apparent transverse strain, i.e. the net transverse displacement, $\Delta L_{\text {TRANS }}$, divided by the specimen width, named apparent since at this stage it still comprises the contribution due to the extensometer knives penetration. Such a representation of the lateral contraction effects, in which also the overall transverse displacement can be easily deduced $\left(\varepsilon_{\text {TRANS }}=\right.$

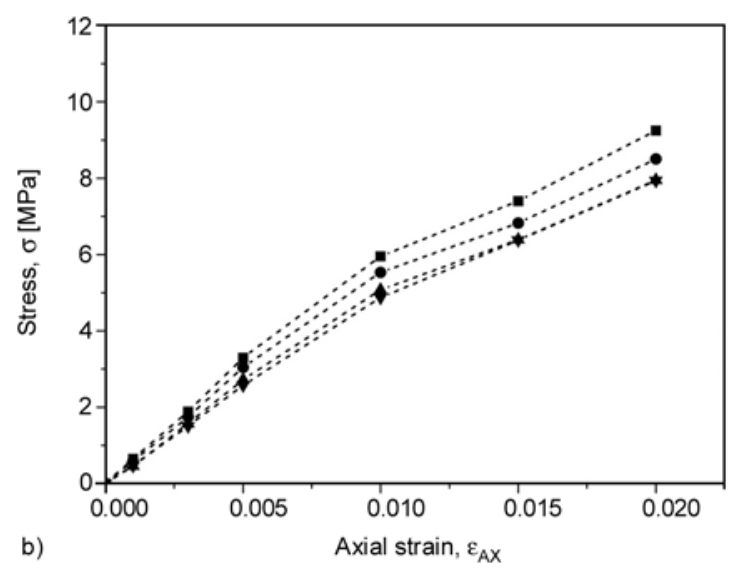

Figure 5. PBT isochronous stress $v s$. strain curves at a) $30^{\circ} \mathrm{C}$ and b) $70^{\circ} \mathrm{C}$ for various instants: $10 \mathrm{~s} \mathrm{( \bullet );} 100 \mathrm{~s}(\bullet) ; 1000 \mathrm{~s}(\boldsymbol{\Delta})$; $1 \mathrm{~h}(\boldsymbol{\nabla})$ 

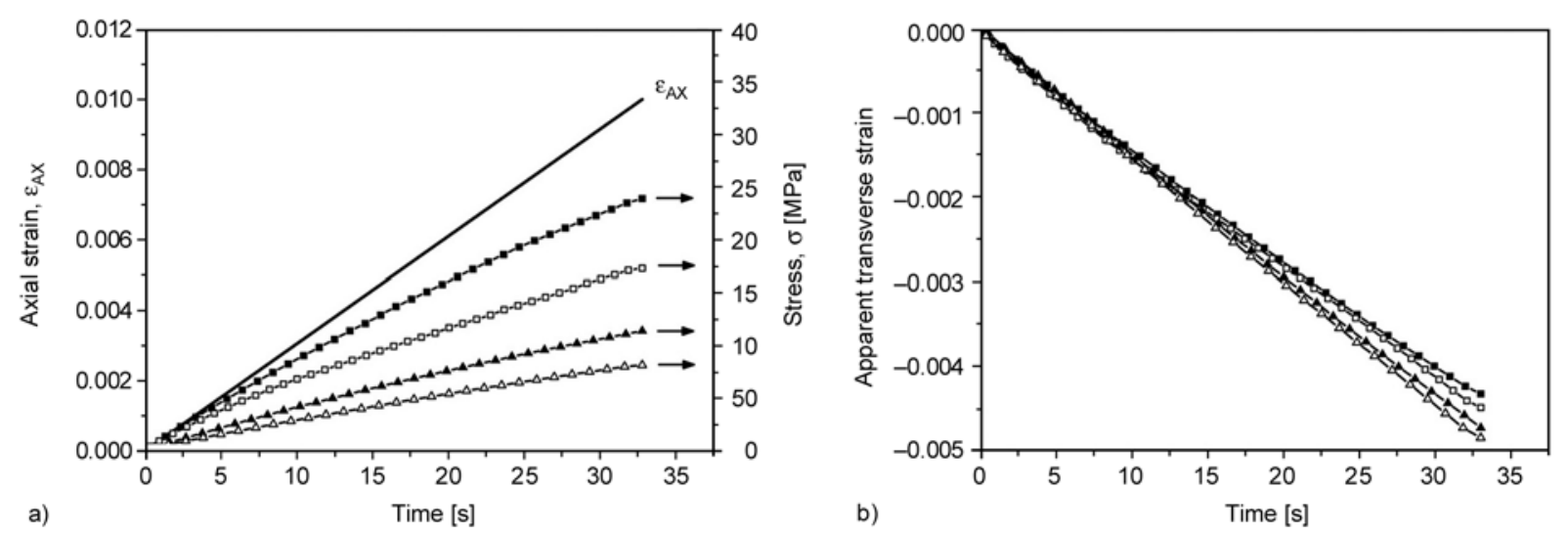

Figure 6. Time evolution of a) axial strain, $\varepsilon_{\mathrm{AX}}$, and stress, $\sigma$, and b) apparent transverse strain in CDR experiments for an axial strain rate of $0.003 \mathrm{~s}^{-1}$ at various temperatures: $20^{\circ} \mathrm{C}(\mathbf{\square}) ; 30^{\circ} \mathrm{C}(\square) ; 50^{\circ} \mathrm{C}(\Delta) ; 60^{\circ} \mathrm{C}(\Delta)$

-0.001 corresponds to a transverse contraction of $10 \mu \mathrm{m}$ ), may help the comparison of the transverse displacement data with the data regarding the extensometer penetration, which will be reported later.

The opposite signs exhibited by axial and transverse strains indicate that the application of a tensile deformation leads to lateral contraction of the specimen, and it is shown that at any given instant, and thus at any given axial strain value, a greater lateral contraction is found as the temperature increases. The total transverse displacement measured within this set of tests is between 40 and $50 \mu \mathrm{m}$, depending on the test temperature.

The input and output of constant deformation tests are reported in Figures $7 \mathrm{a}$ and $\mathrm{b}$ in case of an axial deformation $\varepsilon_{\mathrm{AX}}=0.01$ and at various temperatures. These figures show that the stress relaxation is also accompanied by a continuous lateral contraction process. While the stress relaxation displays the well-known dependence on time and temperature, the transverse strain decreases with time

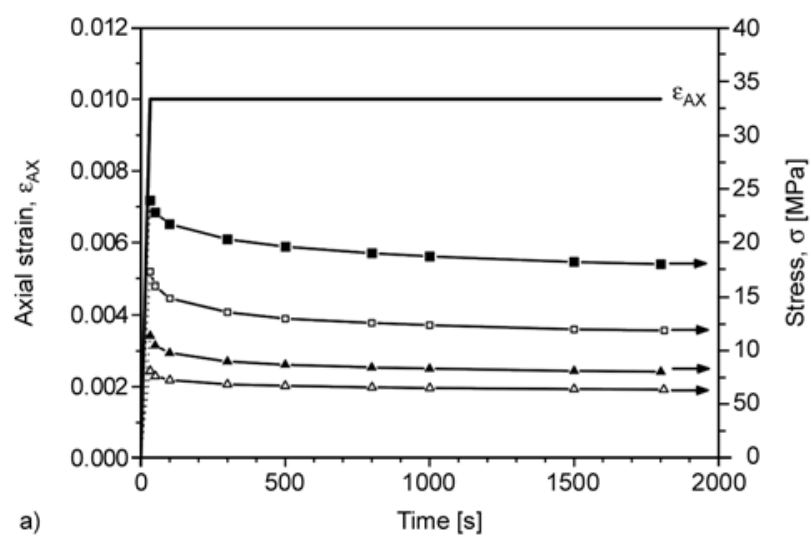

and, at any instant, a greater lateral contraction is found for a higher temperature. Within this set of experiments the change in the net transverse displacement signal during the constant axial strain maintenance is about $5 \mu \mathrm{m}$ at all temperatures, and it is shown that at high temperatures an overlapping of the curves can occur for long times.

In order to correct the value of the transverse displacement for the concomitant lateral creep of the extensometer knives, this latter term was quantitatively estimated in experiments carried out on undeformed specimens. Figure 8 reports some examples of the knives penetration curves. It can be noted that in the time span of CDR tests (i.e. up to $40 \mathrm{~s}$ ), the lateral penetration is in any case lower than $0.1 \mu \mathrm{m}$, and thus very small in comparison with the transverse displacement recorded during the ramp tests; it is therefore believed that the lateral penetration does not practically affect the lateral contraction ratio evaluated in CDR tests. On the other hand, the knives penetration becomes more important for long times, and within $1800 \mathrm{~s}$ a maximum penetra-

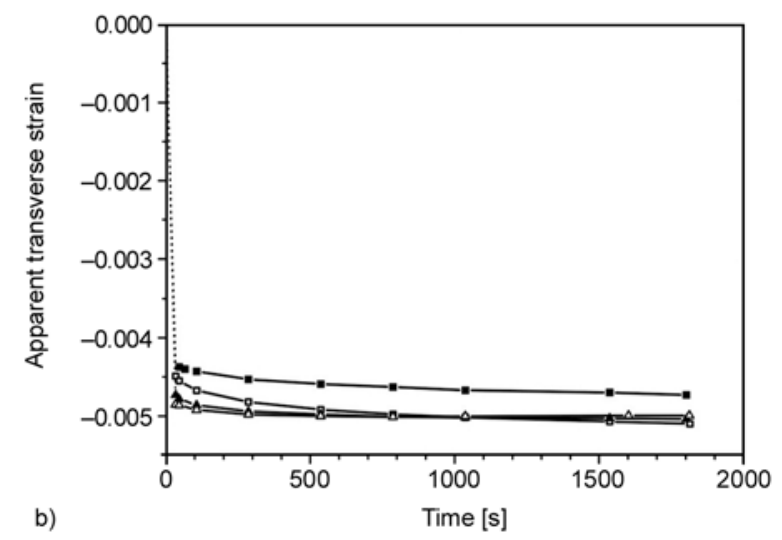

Figure 7. Time evolution of a) axial strain, $\varepsilon_{\mathrm{AX}}$, and stress, $\sigma$, and b) apparent transverse strain in REL experiments carried out at $\varepsilon_{\mathrm{AX}, \mathrm{REL}}=0.01$ at various temperatures: $20^{\circ} \mathrm{C}(\boldsymbol{\square}) ; 30^{\circ} \mathrm{C}(\square) ; 50^{\circ} \mathrm{C}(\boldsymbol{\Delta}) ; 60^{\circ} \mathrm{C}(\Delta)$ 


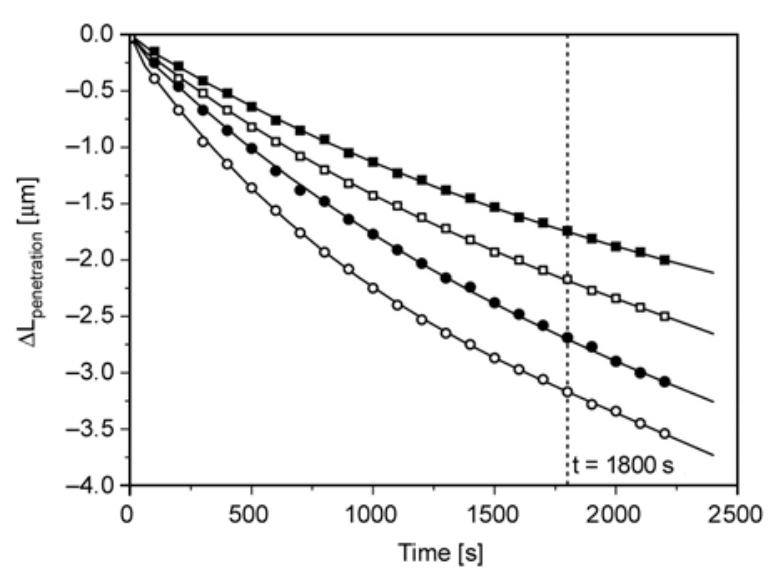

Figure 8. Transverse displacement due to the extensometer lateral penetration measured on an undeformed specimen as a function of time at various temperatures: $30^{\circ} \mathrm{C} \mathrm{( \bullet );} 40^{\circ} \mathrm{C}(\square) ; 50^{\circ} \mathrm{C}(\bullet) ; 60^{\circ} \mathrm{C}(\circ)$

tion of about $1.7 \mu \mathrm{m}$ is found at $30^{\circ} \mathrm{C}$ and almost the double at $60^{\circ} \mathrm{C}$. These values are not negligable if compared with the transverse displacement variation recorded during REL tests. In fact, at the two temperatures considered, they reach about 30 and $60 \%$ of the values measured in REL tests during the constant axial strain maintenance, respectively. This indicates that the compensation of such undesired effect becomes a strict requirement in order to provide a quantitative evaluation of the viscoelastic Poisson's ratio in relaxation tests.

\subsection{Constant deformation rate tests}

The effects of temperature and strain rate on the short-time Poisson's ratio, $v_{\mathrm{CDR}}$, were investigated in CDR experiments. The results of the experiments are reported in Figures 9a and b, representing the Poisson's ratio and Young's modulus, respectively, as a function of temperature at two strain rates.

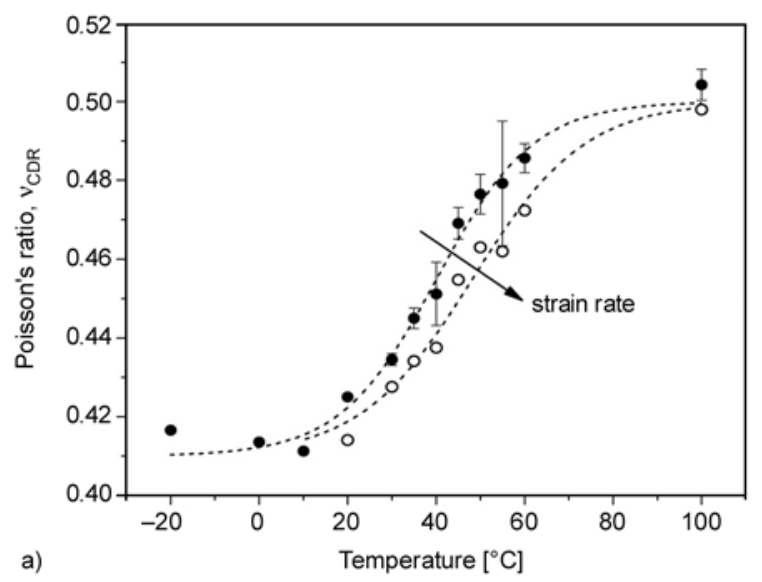

Figure 9a shows that, for both strain rates, the Poisson's ratio increases with temperature. Such dependence can be fitted with a sigmoidal function, extending from a lower plateau value of about 0.41 to an upper plateau value scattered around 0.5 , representative of the conditions of constant volume deformation, typical of incompressible materials in the rubbery state. The inflection point of the sigmoidal curves is located for both strain rates at temperatures in the proximity of the PBT glass transition temperature, whereas the upper plateau is approached at about $100^{\circ} \mathrm{C}$, i.e. well above $T_{\mathrm{g}}$. As the strain rate increases, a lower $v_{\mathrm{CDR}}$ value is found for a given temperature, and a shift of the sigmoidal fitting curve to higher temperatures can be observed. Such a shift, if evaluated with reference to the inflection point, is about $10^{\circ} \mathrm{C}$ for a strain rate increase of about 20 times. The Young's modulus, which is measured in the same experiment, displays a sigmoidal decreasing dependence on temperature, only slightly affected by strain rate. For both strain rates the inflection point of the curves is located at about $40^{\circ} \mathrm{C}$, in proximity to that found for the Poisson's ratio measured at the lower strain rate.

It is interesting to compare these results with those previously obtained by this group, under similar experimental methodology, on epoxy resins [14]. A similar sigmoidal dependence on temperature was evidenced for the epoxy systems, but with an inflection point for the Poisson's ratio vs. temperature curve occurring $20^{\circ} \mathrm{C}$ below the inflection point of the simultaneously measured Young's modulus vs. temperature curve. Moreover, the upper plateau was approached for deformation temperatures close to the glass transition temperature. Tcharkhtchi et al.

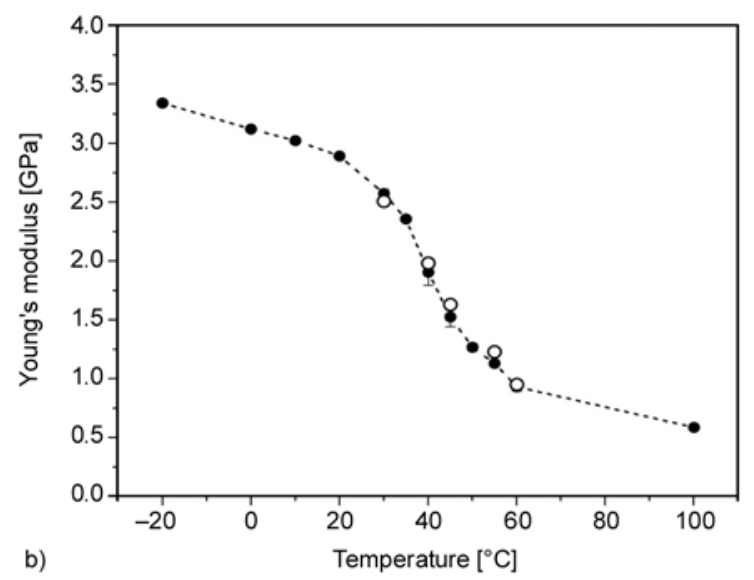

Figure 9. a) Viscoelastic Poisson's ratio, $v_{\mathrm{CDR}}$, and b) Young modulus for semicrystalline PBT evaluated at different deformation temperatures along a deformation ramp at strain rate of $0.003 \mathrm{~s}^{-1}(\bullet)$ and of $0.05 \mathrm{~s}^{-1}(\circ)$ 


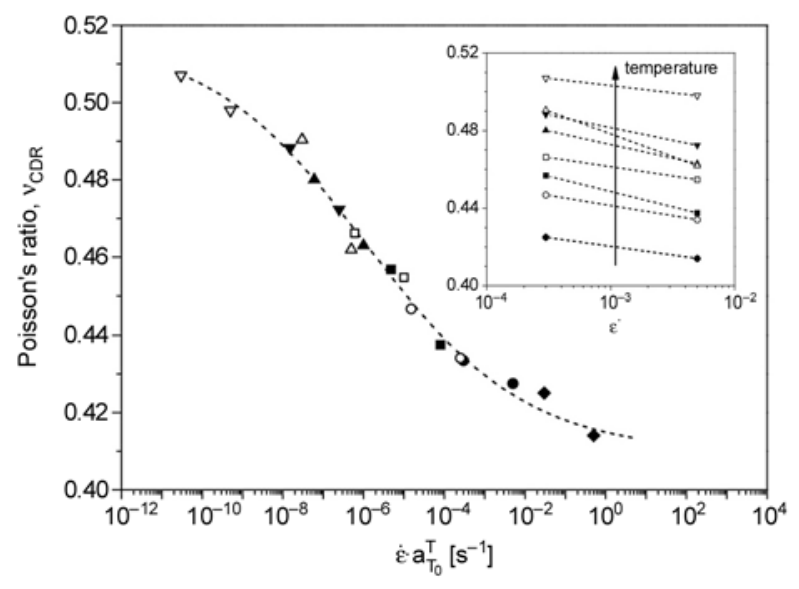

Figure 10. Viscoelastic Poisson's ratio, $v_{\mathrm{CDR}}$, master curve as function of reduced strain rate at a reference temperature $T_{0}=30^{\circ} \mathrm{C}$ after shifting of the isothermal curves at temperatures: $20^{\circ} \mathrm{C}(\downarrow)$;

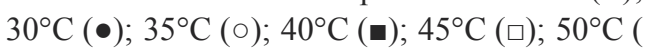
$\Delta) ; 55^{\circ} \mathrm{C}(\Delta) ; 60^{\circ} \mathrm{C}(\boldsymbol{\nabla}) ; 100^{\circ} \mathrm{C}(\nabla)$; insert: isothermal curves before shifting

[15] provided analogous results on similar epoxy systems. The results here presented for semicrystalline PBT suggest that higher temperatures are required with respect to amorphous materials to display the quasi-constant volume conditions and that the $T_{\mathrm{g}}$ region plays an important role on the transition to such deformational behaviour. This result could be interpreted by considering a mobility hindrance exerted by the coexistence of crystalline and amorphous regions.

By a tentative application of a time-temperature reduction scheme, the isothermal $v_{\mathrm{CDR}}$ data, represented as a function of the strain rate, were rigidly shifted along the strain rate axis until the best superposition was reached. The master curve obtained is presented for a reference temperature $T_{0}=30^{\circ} \mathrm{C}$ in Figure 10. The decreasing (sigmoidal) dependence of the Poisson's ratio on strain rate indicates that as the strain rate decreases the deformation can occur under quasi-constant volume conditions, and, although only qualitatively, the result seems to suggest that for a deformation at $30^{\circ} \mathrm{C}$ these deformation conditions can be met for very slow strain rates, lower than about $10^{-9} \mathrm{~s}^{-1}$.

\subsection{Constant deformation tests}

REL tests permitted the investigation of Poisson's ratio dependence on time and temperature, starting from the net transverse displacement signal and correcting it for the extensometer knives penetra- tion. The results obtained are reported in Figure 11a, which displays the Poisson's ratio, $v_{\mathrm{REL}}$, as a function of the relaxation time, $t_{\mathrm{REL}}$, in terms of isothermal curves measured for an applied axial deformation $\varepsilon_{\mathrm{AX}}=0.01$. For all the investigated temperatures, the viscoelastic Poisson's ratio displays a monotonic increase with time, and, for any given instant, a higher value of the Poisson's ratio is found as temperature increases. The average slope of the curves increases for temperatures up to $40^{\circ} \mathrm{C}$, i.e. in the proximity of the glass transition temperature, and decreases again for temperatures above $T_{\mathrm{g}}$.

The initial part of the curves is characterized by a fairly regular increase, whereas at longer times $\left(t_{\mathrm{REL}}\right.$ longer than approximately $10 \mathrm{~min}$ ) the isothermal curves tend to overlap, exhibiting a less regular behaviour and the tendency to converge to a value slightly above 0.5 , i.e. above the incompressibility conditions. It is important to underline that if the transverse extensometer reading was not corrected, even higher Poisson's ratio values would have been read. In the author's opinion these high Poisson's ratio values have to be interpreted as scattered around 0.5 , and affected by inaccuracies related to the correction of transverse deformation for long times and high temperatures. At the same time, it can not be excluded that the upper plateau could be higher than 0.5 , due to non-homogeneity on a microstructural scale, already present in the materials (such as crystalline regions below the melting temperature [1] or porosity [28]) or developed during the deformational process (as cavitational effects [29-32], although these should have major importance at deformations higher than those investigated here).

The decrease of stress was simultaneously monitored, permitting an evaluation of the time evolution of the relaxation modulus $E_{\mathrm{REL}}$, which is reported in Figure 11b in terms of isothermal curves for an axial deformation $\varepsilon_{\mathrm{AX}}=0.01$. The well-known dependence on time and temperature is exhibited and it is noteworthy to underline that the steepest decrease is found at $T=40^{\circ} \mathrm{C}$.

As previously shown in the evaluation of short-time Poisson's ratio by CDR tests, the glass transition temperature seems to play a relevant role on the PBT viscoelastic Poisson's ratio also even under these loading conditions. Not being directly in possession of data regarding the effect of temperature 

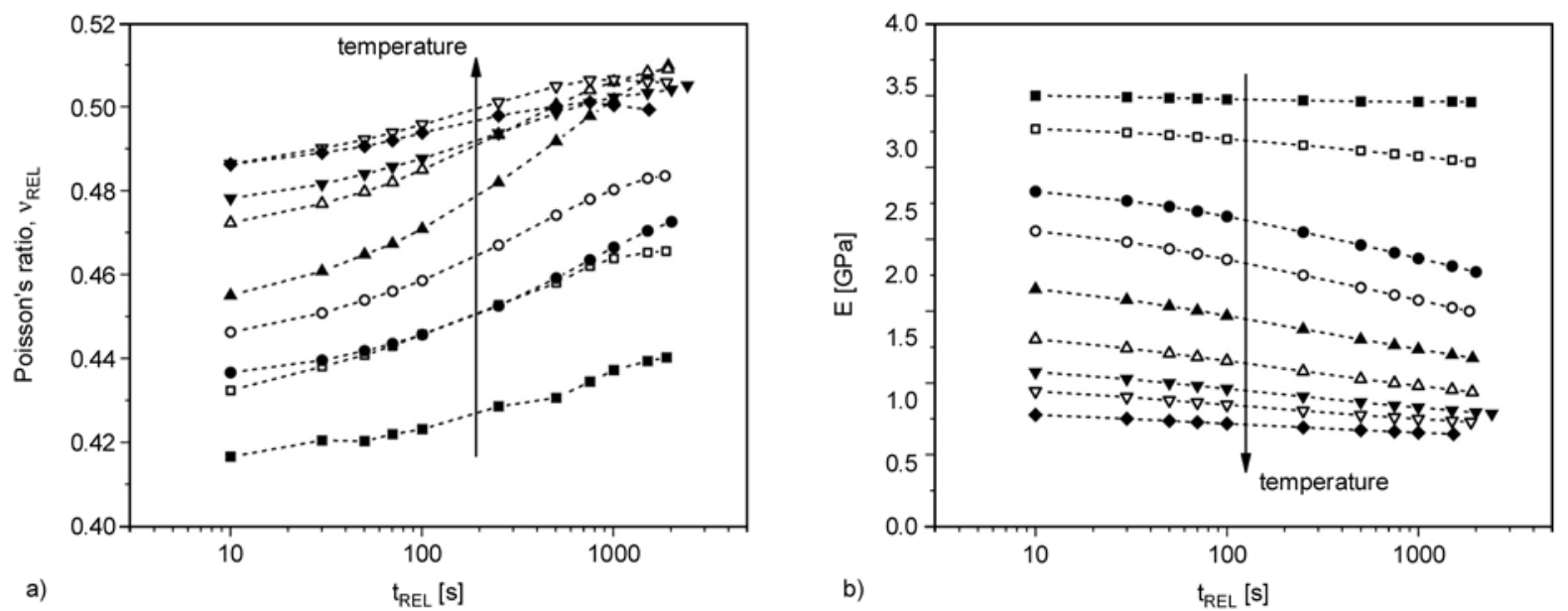

Figure 11. a) Isothermal Poisson's ratio, $v_{\mathrm{REL}}$, and b) relaxation modulus, $E$, isothermal curves as a function of relaxation time, $t_{\mathrm{REL}}$, at various temperatures: $0^{\circ} \mathrm{C}(\boldsymbol{\bullet}) ; 20^{\circ} \mathrm{C}(\square) ; 30^{\circ} \mathrm{C}(\bullet) ; 35^{\circ} \mathrm{C}(\circ) ; 40^{\circ} \mathrm{C}(\boldsymbol{\Delta}) ; 45^{\circ} \mathrm{C}(\Delta) ; 50^{\circ} \mathrm{C}(\boldsymbol{\nabla})$; $55^{\circ} \mathrm{C}(\nabla) ; 60^{\circ} \mathrm{C}(\diamond)$; data obtained for an axial deformation $\varepsilon_{\mathrm{AX}, \mathrm{REL}}=0.01$

on relaxation Poisson's ratio of amorphous materials, our results were compared with literature data, which underline some relevant differences between semicrystalline and amorphous polymers. O'Brien et al. [17] reported isothermal curves of the viscoelastic so-called lateral contraction ratio for epoxy resins under creep conditions. They evidenced an upper plateau at 0.49 for temperatures approaching the glass transition region, while the simultaneously measured creep compliance still displays an increasing trend. Similar results were obtained on amorphous poly (methylmetacrylate) by the group of Knauss [10]. In both the above-cited works the steepest part of the Poisson's ratio isothermal curve is located at temperatures lower than $T_{\mathrm{g}}$.

The REL experiments performed at $\varepsilon_{\mathrm{AX}}=0.005$ led to results similar to those obtained at the higher deformation level, although a less regular trend for the isothermal Poisson's ratio vs. relaxation time curves was found. This is probably due to the fact that, in the case of a lower axial deformation, the extensometer knives penetration becomes of higher importance with respect to the transverse contraction, and thus an even greater accuracy is required for the data correction.

On the basis of a time-temperature reduction scheme, the $v_{\mathrm{REL}}$ and $E_{\mathrm{REL}}$ isothermal curves obtained for the two sets of axial strains were shifted along the time scale, in order to tentatively achieve a master curve representation of the two functions. The master curves obtained are reported in Figures 12a and b, in which the Poisson's ratio and the relaxation modulus, respectively, are plotted as a function of the reduced time for a reference temperature $T_{0}=30^{\circ} \mathrm{C}$; Figure $12 \mathrm{~b}$ reports also the Poisson's ratio master curve evaluated at $\varepsilon_{\mathrm{AX}}=0.01$ in order to directly compare the time dependence of the two functions.
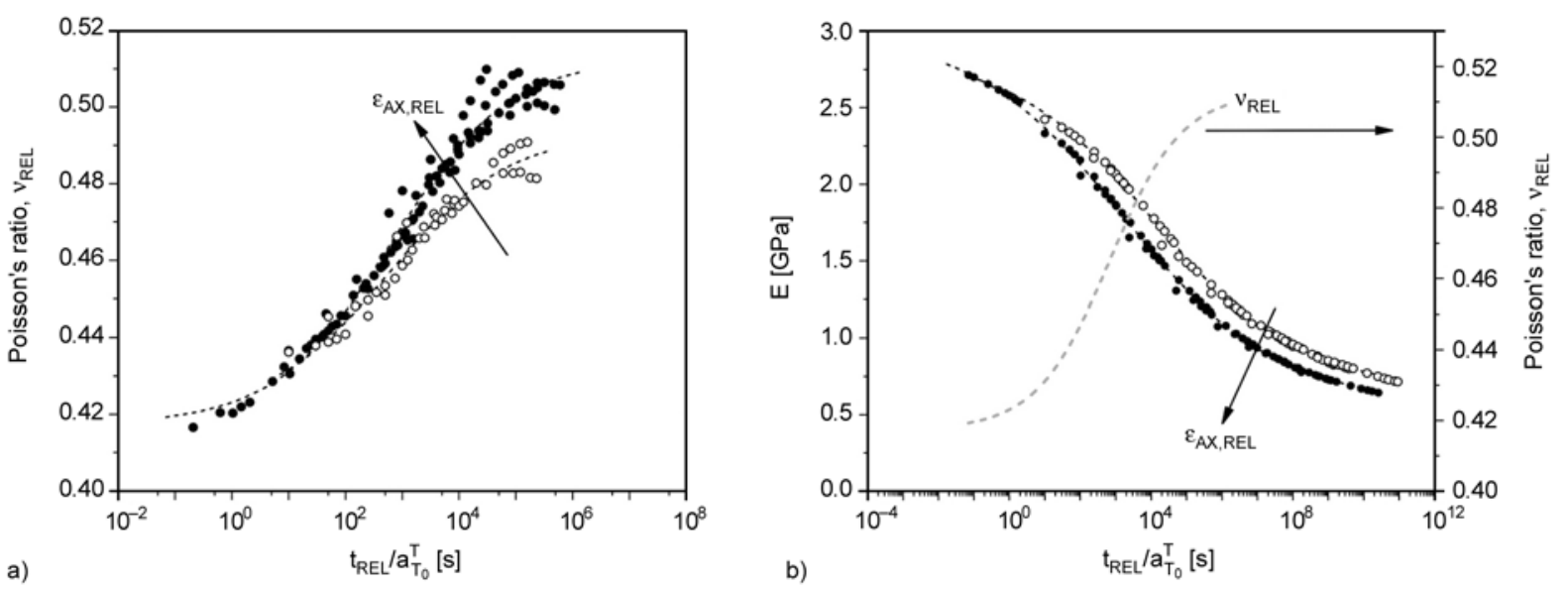

Figure 12. a) Poisson's ratio, $v_{\mathrm{REL}}$, and b) relaxation modulus, $E$, master curves as a function of reduced relaxation time at a reference temperature $T_{0}=30^{\circ} \mathrm{C}$ for two different axial deformations $\varepsilon_{\mathrm{AX}, \mathrm{REL}}: 0.005(\mathrm{O})$ and $0.01(\bullet)$ 
For both axial strain levels, Poisson's ratio shows an increasing trend with time, which can be fitted by a sigmoidal function and can be viewed as a retardation (or delay) process. At this reference temperature the master curve upper plateau seems to be approached at times in the order of $10^{6} \mathrm{~s}$ and the inflection point is located at about $10^{3} \mathrm{~s}$. The applied axial strain seems to marginally affect the time-dependence of the viscoelastic Poisson's ratio, and mainly in the region of longer relaxation times: in fact, although following similar sigmoidal trend, higher Poisson's ratio values can be found for a given instant as the applied strain increases. Also the relaxation modulus master curve seems to be dependent on the applied strain, and lower relaxation moduli are found at the higher strain. Such effect on both viscoelastic functions is interpreted as a typical non-linear viscoelastic effect related to the higher mobility occurring when larger deformations are applied.

Several aspects anyway suggest that the time distribution of the Poisson's ratio master curve here provided, at this stage, should be considered as merely qualitative; these aspects regard i) the different distribution of the Poisson's ratio and of the relaxation modulus as a function of the reduced time scale, and ii) the different shift-factors for the two functions.

In Figure $12 \mathrm{~b}$ it is shown that, with respect to $E_{\mathrm{REL}}$, the $v_{\text {REL }}$ function displays inflection points occurring at shorter times (the $E_{\mathrm{REL}}$ inflection point being at about $10^{6} \mathrm{~s}$ ) and a narrower transition region. This latter is seen to span over 3-4 decades in the case of $v_{\mathrm{REL}}$, thus on a significantly narrower region with respect to the 7-8 decades transition region exhibited by the simultaneously measured relaxation modulus, and, similarly, by the storage modulus master curve. Since Tschoegl et al. [1] claims that the delay times distribution can not be assumed to be identical to that of relaxation and retardation times measured in stress relaxation and creep experiments, such a result can not be regarded as inconsistent. Nevertheless, inspection of literature data does not provide great help in interpreting this effect. A tentative explanation of the peculiar distribution of delay times observed for PBT's $v_{\mathrm{REL}}$ with respect to relaxation times shown by $E_{\mathrm{REL}}$ could be based on the different relaxation kinetics of the hydrostatic and deviatoric stress components of the stresses. In fact, it was experimentally proven that bulk relaxation shows a narrower distribution of relaxation times with respect to shear relaxation (Morita et al. on poly (isobutyl metacrylate), and Kono et al. on poly (isobutylether); both listed in [1]); further, Di Landro and Pegoraro [18] have attributed to the same reason the observed transition of the viscoelastic Poisson's ratio of polyetherimide (PEI) occurring at times shorter than that measured for shear and tensile modulus.

Of more difficult interpretation is the result concerning the different shift factors required for the construction of Poisson's ratio ( $v_{\mathrm{CDR}} v s$. strain rate; $v_{\text {REL }} v s$. time) and of the relaxation and storage moduli master curves, as can be seen from their representation as a function of the inverse absolute temperature in Figure 13. The shift factors of the relaxation modulus are not affected by the strain level and exhibit values quite close to those estimated in DMTA tests. The shift factors for the master curves of Poisson's ratio in REL tests also seem to be unaffected by the axial strain, but show a less steep slope with respect to those related to the moduli functions. Finally, the data obtained from the Poisson's ratio measured in CDR experiments, shows an intermediate behavior, exhibiting values close to those measured for the moduli functions for temperature up to $40^{\circ} \mathrm{C}$, while a deviation is exhibited for higher temperatures, with a slope similar to that shown by the $v_{\text {REL }}$ set of data. Previous literature data have always shown that the shift factors

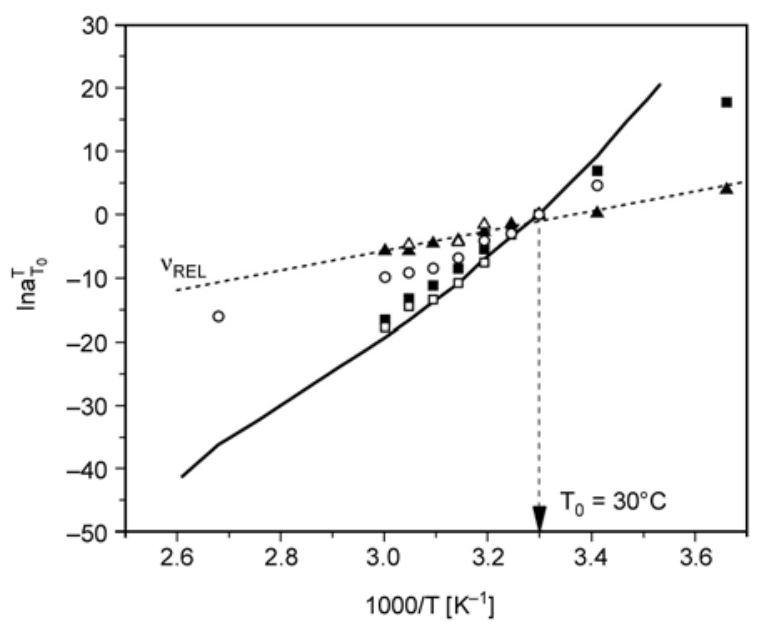

Figure 13. Shift factors employed for the construction of the following master curves: a) storage modulus vs. frequency, b) Poisson's ratio $v_{\mathrm{CDR}}$ vs. strain rate, c) Poisson's ratio $v_{\mathrm{REL}} v s . t_{\mathrm{REL}}\left(\varepsilon_{\mathrm{AX}, \mathrm{REL}}=\right.$ $0.005(\mathbf{\Delta})$ and $0.01(\mathbf{\square}))$ and $E v s . t_{\mathrm{REL}}\left(\varepsilon_{\mathrm{AX}, \mathrm{REL}}=\right.$ $0.005(\Delta)$ and $0.01(\square))$ 
required for the Poisson's ratio master curve construction are the same as those obtained for other viscoelastic functions $[10,17,20]$. The difference found between master curves distribution and between the related shift factors are effects not fully understood, and that could be tentatively ascribed to an interference due to the contact extensometer. In fact, although the subtraction of the transverse penetration is believed to correct the value of Poisson's ratio in any experimental condition, the presence of additional stress components, locally induced by the lateral clip-on device, cannot be avoided.

\section{Conclusions}

The viscoelastic dependence of Poisson's ratio on time and temperature was investigated for semicrystalline PBT in tensile ramp tests at constant deformation rate (CDR tests) and in tests in which the axial strain was maintained constant (constant deformation, or REL, tests). The axial and transverse deformations were simultaneously recorded by means of a biaxial clip-on extensometer, and a correction procedure was proposed in order to subtract the lateral penetration of the extensometer knives from the net transverse displacement recorded.

It was shown that PBT Poisson's ratio exhibits viscoelastic features, with values increasing with time and temperature and decreasing with strain rate. The results of CDR tests show that the short-time Poisson's ratio, $v_{\mathrm{CDR}}$, measured at strain rates in the order of $10^{-3}-10^{-2} \mathrm{~s}^{-1}$, displays a sigmoidal increase with temperature with an upper plateau at about 0.5 (i.e. the constant volume deformation conditions), approached for temperatures well above $T_{\mathrm{g}}$, in contrast to what was reported for amorphous polymers, which generally reach such deformational behaviour at $T_{\mathrm{g}}$. Further, for the same temperature a higher $v_{\mathrm{CDR}}$ value is found as the strain rate increases, and a tentative representation of the $v_{\mathrm{CDR}} v s$. strain rate master curve would suggest that, for a reference temperature $T_{0}=30^{\circ} \mathrm{C}$, the upper plateau can be approached for strain rates lower than $10^{-9} \mathrm{~s}^{-1}$.

In REL tests it is shown that stress relaxation is accompanied by a continuous lateral contraction process, which leads to a monotonous increase of Poisson's ratio, $v_{\text {REL }}$, with time, exhibiting the fastest increase in proximity to the glass transition temperature. The master curve representation of Poisson's ratio, tentatively obtained from the isothermal $v_{\mathrm{REL}}$ curves, qualitatively describes a sigmoidal increasing dependence on the logarithmic reduced time, confirming the nature of viscoelastic Poisson's ratio as a retardation (or delay) process.

\section{References}

[1] Tschoegl N. W., Knauss W. G., Emri I.: Poisson's ratio in linear viscoelasticity - A critical review. Mechanics of Time-Dependent Materials, 6, 3-51 (2002). DOI: 10.1023/A:1014411503170

[2] Hilton H. H.: Implications and constraints of timeindependent Poisson ratios in linear isotropic and anisotropic viscoelasticity. Journal of Elasticity, 63, 221-251 (2001). DOI: $10.1023 / \mathrm{A}: 1014457613863$

[3] Lakes R. S., Wineman A.: On Poisson's ratio in linearly viscoelastic solids. Journal of Elasticity, 85, 4563 (2006).

DOI: $10.1007 / \mathrm{s} 10659-006-9070-4$

[4] Hilton H. H., Yi S.: The significance of (an)isotropic viscoelastic Poisson ratio stress and time dependencies. International Journal of Solids and Structures, 35, 3081-3095 (1998).

DOI: $10.1016 / \mathrm{S} 0020-7683(97) 00357-0$

[5] Delin M., Rychwalski W. R., Kubát J. M., Kubát J.: Volume changes during stress relaxation in polyethylene. Rheologica Acta, 34, 182-195 (1995).

DOI: $10.1007 / \mathrm{BF} 00398438$

[6] Bertilsson H., Delin M., Kubát J., Rychwalski W. R., Kubát M. J.: Strain rates and volume changes during short-term creep of PC and PMMA. Rheologica Acta, 32, 361-369 (1993). DOI: $10.1007 / \mathrm{BF} 00435082$

[7] Tsou A. H., Greener J., Smith G. D.: Stress relaxation of polymer films in bending. Polymer, 36, 949-954 (1995).

DOI: 10.1016/0032-3861(95)93593-B

[8] Drozdov A. D.: Volume changes in glassy polymers. Archive of Applied Mechanics, 68, 689-710 (1998). DOI: $10.1007 / \mathrm{s} 004190050197$

[9] Popelar C. F., Liechti K. M.: A distortion-modified free volume theory for nonlinear viscoelastic behavior. Mechanics of Time-Dependent Materials, 7, 89-141 (2003).

DOI: 10.1023/A:1025625430093

[10] Lu H., Zhang X., Knauss W. G.: Uniaxial, shear, and Poisson relaxation and their conversion to bulk relaxation: Studies on poly(methyl methacrylate). Polymer Engineering and Science, 37, 1053-1064 (1997). DOI: $10.1002 /$ pen. 11750

[11] Sane S. B., Knauss W. G.: The time-dependent bulk response of poly (methyl methacrylate). Mechanics of Time-Dependent Materials, 5, 325-343 (2001). DOI: 10.1023/A:1012517421899 
[12] Okoli O. I., Smith G. F.: The effect of strain rate and fibre content on the Poisson's ratio of glass/epoxy composites. Composite Structures, 48, 157-161 (2000). DOI: 10.1016/S0263-8223(99)00089-6

[13] Steinberger R., Jerabek M., Major Z., Lang R. W.: Determination of time dependent Poisson's ratio vales of polypropylene in monotonic and creep tensile experiments. in: 'Proceedings of the $13^{\text {th }}$ International Conference on Deformation, Yield and Fracture of Polymers, Kerkrade, The Netherlands' 381-384 (2006).

[14] Pandini S., Pegoretti A.: Time, temperature, and strain effects on viscoelastic Poisson's ratio of epoxy resins. Polymer Engineering and Science, 48, 1434-1441 (2008).

DOI: $10.1002 /$ pen.21060

[15] Tcharkhtchi A., Faivre S., Roy L. E., Trotignon J. P., Verdu J.: Mechanical properties of thermosets. Journal of Materials Science, 31, 2687-2692 (1996). DOI: $10.1007 / \mathrm{BF} 00687301$

[16] Jazouli S., Luo W., Brémand F., Vu-Khanh T.: Nonlinear creep behavior of viscoelastic polycarbonate. Journal of Materials Science, 41, 531-536 (2006). DOI: $10.1007 / \mathrm{s} 10853-005-2276-1$

[17] O’Brien D. J., Sottos N. R., White S. R.: Cure-dependent viscoelastic Poisson's ratio of epoxy. Experimental Mechanics, 47, 237-249 (2007).

DOI: $10.1007 / \mathrm{s} 11340-006-9013-9$

[18] Di Landro L., Pegoraro M.: Determinazione delle funzioni viscoelastiche di materiali polimerici (in Italian). in: 'Proceedings of IV National Congress AIMAT, Cagliari, Italy’ 663-668 (1998).

[19] Caracciolo R., Giovagnoni M.: Frequency dependence of Poisson's ratio using the method of reduced variables. Mechanics of Materials, 24, 75-85 (1996). DOI: 10.1016/0167-6636(96)00002-6

[20] Caracciolo R., Gasparetto A., Giovagnoni M.: An experimental technique for complete dynamic characterization of a viscoelastic material. Journal of Sound and Vibration, 272, 1013-1032 (2004).

DOI: $10.1016 /$ j.jsv.2003.03.008

[21] Arzoumanidis G. A., Liechti K. M.: Linear viscoelastic property measurement and its significance for some nonlinear viscoelasticity models. Mechanics of TimeDependent Materials, 7, 209-250 (2003). DOI: 10.1023/B:MTDM.0000007357.18801.13
[22] Litt M. H., Torp S.: Strain softening and yeild of polycarbonate-Moiré-grid biaxial-strain analysis. Industrial and Engineering Chemistry Product Research and Development, 11, 357-363 (1972).

DOI: $10.1021 / 1360043 a 021$

[23] Kästner S., Pohl G.: Ein Beitrag zur Frage der vollständigen Erfassung des mechanischen Relaxationsverhaltens der Polymeren. Colloid and Polymer Science, 191, 114-123 (1963).

[24] Pritz T.: The Poisson's loss factor of solid viscoelastic materials. Journal of Sound and Vibration, 306, 790802 (2007). DOI: $10.1016 /$ j.jsv.2007.06.016

[25] Zhang L., Ernst L. J., Brouwer H. R.: A study of nonlinear viscoelasticity of an unsaturated polyester resin. Part 2. 3D model. Mechanics of Materials, 26, 167195 (1997). DOI: 10.1016/S0167-6636(97)00029-X

[26] Lakes R. S.: The time-dependent poissons ratio of viscoelastic cellular materials can increase or decrease. Cellular Polymers, 11, 466-469 (1992).

[27] van Krevelen D. W.: Properties of polymers. Elsevier, Amsterdam (1990).

[28] Pawlak A., Galeski A.: Plastic deformation of crystalline polymers: The role of cavitation and crystal plasticity. Macromolecules, 38, 9688-9697 (2005). DOI: $10.1021 / \mathrm{ma} 0508420$

[29] Pawlak A., Galeski A.: Cavitation during tensile deformation of polypropylene. Macromolecules, 41, 28392851 (2005). DOI: $10.1021 / \mathrm{ma} 0715122$

[30] Quatravaux T., Elkoun S., G'Sell C., Cangemi L., Meimon Y.: Experimental characterization of the volume strain of poly(vinylidene fluoride) in the region of homogeneous plastic deformation. Journal of Polymer Science Part B: Polymer Physics, 40, 2516-2522 (2002).

DOI: $10.1002 /$ polb.10318

[31] Addiego F., Dahoun A., G'Sell C., Hiver J. M.: Volume variation process of high-density polyethylene during tensile and creep tests. Oil and Gas Science and Technology - Rev. IFP, 61, 715- 724 (2006). DOI: 10.2516/ogst:2006009

[32] Addiego F., Dahoun A., G'Sell C., Hiver J-M.: Characterization of volume strain at large deformation under uniaxial tension in high-density polyethylene. Polymer, 47, 4387-4399 (2006).

DOI: $10.1016 /$ j.polymer.2006.03.093 